\section{(A) Check for updates}

Cite this: Food Funct., 2021, 12, 7185

\title{
Improving the nutritional profile of culinary products: oleogel-based bouillon cubes
}

\author{
Valentina Conty, (D) * Sophia Theierl and Eckhard Flöter
}

\begin{abstract}
Structured fat phases are the basis of many consumer relevant properties of fat-containing foods. To realise a nutritional improvement - less saturated, more unsaturated fatty acids - edible oleogels could be remedy. The feasibility of traditional fat phases structured by oleogel in culinary products has been evaluated in this study. In this contribution the oleogel application in bouillon cubes as model system for culinary products is discussed. Three different gelators (sunflower wax (SFW), a mixture of $\beta$-Sitosterol and $\gamma$-Oryzanol (SO) and ethylcellulose (EC)), at two concentration levels $(5 \%$ and $10 \%(\mathrm{w} / \mathrm{w})$ ) each, were evaluated with respect to their physical properties, in the food matrix and application. The application of pure and structured canola oil (CO) was benchmarked against the reference, palm fat (PO). The assessment of the prototypes covered attempts to correlate the physicochemical analyses and sensory data. Organoleptic and analytical studies covered storage stability (up to 6 months) monitoring texture, color and fat oxidation. The results indicate that the substitution of palm fat by oleogel is essentially possible. The characteristics of the bouillon cubes are tuneable by gelator choice and inclusion level. Most importantly, the data show that the anticipated risk of intolerable effects of oxidation during shelf life is limited if antioxidants are used
\end{abstract}

Received 22nd May 2021, Accepted 21st June 2021 DOI: $10.1039 /$ d1fo01589c rsc.li/food-function properties to provide crucial functionalities in food. However, if techno-functional food components are varied, as in fat substitution, this has an effect on the entire product: the manufacturing process, sensory properties (appearance/color, mouthfeel and taste), oil separation, melting behaviour as well as product shelf life and stability of the food.

The use of oleogels, lipid phases structured by other means than saturated triglycerides, is beneficial for almost all sectors of the food industry, where considerable amounts of hardstock fats are applied. This is clearly demonstrated by the large number of research projects on potential product applications aiming at completely or partially replacing the high SaFA fats currently used. These include baked goods, ${ }^{5}$ dairy products, ${ }^{6-8}$ spreads ${ }^{9}$ and confectionery ${ }^{10}$ as well as meat products. ${ }^{11-13}$ It is noticeable that no publications exist so far in the field of culinary products, although the fat content in these products is typically around $20 \%$.

Bouillon cubes became popular worldwide after the First World War to compensate the lack of micronutrients. ${ }^{14}$ Over the past decades, the image among consumers in the industrialised world has changed considerably as a result of the general improvement in living conditions. ${ }^{14}$ Whereas they play a central role in West and Central Africa, where bouillon cubes are consumed 5-7 days a week in $80-99 \%$ of all households. ${ }^{15-20}$ For this reason, a large number of studies have already investigated the function of bouillon cubes as a carrier for micronutrient fortification, as bouillon cubes are a 
promising carrier due to their simple production process and composition. ${ }^{16,19-21}$ This usually involves mixing all the dry ingredients (salt, possibly glutamate, sugar and herbs) with molten high melting fats (refined, mostly hydrogenated, vegetable oils or animal fats). The hot mixture is shaped, wrapped and packaged after cooling processes that yield the desired consistency. ${ }^{21}$ The solid fats function primarily as a binder immobilizing the dry ingredients in the matrix. The type of fat impacts the sensory properties of the bouillon cube, but also physico-chemical properties like melting behaviour, macroscopic hardness and their resistance to oxidation. ${ }^{14}$ Earlier studies evaluated different commercial bouillon cubes. It was reported that the composition of the fat phases differed significantly. The sensitivity to oxidation corresponded expectedly with the fatty acid compositions. ${ }^{22,23}$ Consequently, the substitution of the currently widely applied fats, being either fully or partially hydrogenated, is a meaningful goal. In addition to the traditional delivery of micronutrients this would yield bouillon cubes with nutritionally improved fatty acid profiles. In this contribution, we report to our knowledge the first time on an attempt to substitute hardstock fats in bouillon cubes with non-triglyceride structured oils. For this purpose, the application of three oleo-gelators with different gelling mechanisms were investigated. Sunflower wax (SFW) is known to form a colloidal crystalline network due to high melting wax esters with agglomerate-sizes up to $40 \mu \mathrm{m} .^{24,25}$ The mixture of $\beta$-Sitosterol and $\gamma$-Oryzanol (SO) assemble into a self-organised fibrillar network which immobilizes the oil. ${ }^{26,27}$ Thirdly, ethylcellulose (EC) forms a polymer network that entraps the liquid oil. ${ }^{28}$ In the field of oleogelation many scientific contributions unfortunately omit evaluations on shelf-life stability. ${ }^{29}$ In the case of bouillon cubes, the fat phase is of particular importance with respect to shelf-life stability since stability against oxidation and oil-leakage are key quality parameters. This is particularly so due to long and rather uncontrolled storage life. To this end lab-scale prototype products were evaluated against reference products over a period of six months.

\section{Experimental}

\section{Materials}

Refined canola oil (CO), called BRÖLIO, was provided by Brökelmann + Co - Oelmühle GmbH + Co (Hamm, Germany) and palm fat (PO) was provided by ADM Hamburg AG (Hamburg, Germany). Sunflower wax (6607L) was supplied by Kahlwax GmbH \& Co KG (Trittau, Germany). Ethylcellulose (Ethocel Standard 100) was obtained from DuPont $\mathrm{GmbH}$ (Lucerne, Switzerland). $\beta$-Sitosterol was received from Acros Organics (New Jersey, USA) and $\gamma$-Oryzanol from IMCD Deutschland GmbH \& Co KG (Cologne, Germany). For the production of bouillon cubes iodized and fluoridated salt (Zentrale Handelsgesellschaft-ZHG-mbH, Offenburg, Germany), fine sugar (Südzucker AG, Mannheim, Germany), Monosodiumglutamate (Umami Seasoning; Ajinomoto Foods Europe Sas, Paris, France), a herb mixture (Mamsell
Salatkräuter; 1 plus-Produkte Handelsgesellschaft $\mathrm{mbH}$, Greven, Germany) and vitamin E (Vitamin E Acetat 97\%; Alfa Aesar; Kandel; Deutschland) were used. All ingredients were used without further modification.

\section{Preparation of oleogels}

Oleogels based on CO and different gelators (SFW, SO and EC) were prepared. All gelator systems were studied at 5\% and 10\% $(\mathrm{w} / \mathrm{w})$ inclusion levels in the lipid phase. In general, the COgelator mixtures were heated up to and kept at $90{ }^{\circ} \mathrm{C}$ for $15 \mathrm{~min}$ to ensure complete dissolution. According to earlier studies EC needs to be dissolved at temperatures above the glass transition temperature and was hence dissolved at $160{ }^{\circ} \mathrm{C}^{10}$ All samples were agitated with a magnetic stirrer at $200 \mathrm{rpm}(400 \mathrm{ml}$ in a glass beaker, heating plate (MR HeiTech, Heidolph Instruments GmbH \& Co.KG, Schwabach)). Subsequently, samples of $50 \pm 1 \mathrm{~g}$ were prepared in preheated $\left(95^{\circ} \mathrm{C}\right)$ Petri dishes by pouring the solution in it. To allow controlled sol-gel transitions the samples were stored for $48 \mathrm{~h}$ at $20 \pm 1.5^{\circ} \mathrm{C}$.

\section{Preparation of bouillon cubes}

The bouillon cubes were composed of sodium chloride $(50 \%)$, fat phase $(20 \%)$, caramelised sugar $(13 \%)$, glutamate $(10 \%)$, mixture of herbs $(7 \%)$ and vitamin $\mathrm{E}(0.01 \%)$. This corresponds to the composition of the conventional bouillon cubes available in supermarkets. To process the ingredients in the laboratory, all solid components were ground to a fine powder in a blender. Table 1 shows the compositions of the fat phases studied. The fat phases of the different samples are composed of either pure palm fat (PO-Ref), straight CO or CO plus a gelator $(90: 10$ or $95: 5 \mathrm{w} / \mathrm{w})$.

For the oleogel-products, the $\mathrm{CO}$ was heated up to $95{ }^{\circ} \mathrm{C}$ for SFW- and SO-based cubes. When applying EC again a temperature of $160{ }^{\circ} \mathrm{C}$ was necessary to dissolve above the glass transition temperature. The lipid phases for cubes based on either straight PO or $\mathrm{CO}$ were kept at a temperature of $50{ }^{\circ} \mathrm{C}$. All samples were stirred with a magnetic stirrer at $200 \mathrm{rpm}$ until the structuring system was completely dissolved. In the next step sodium chloride, glutamate, caramelized sugar and herbs were added under continuous stirring and at the respective constant temperatures ( 90 or $50{ }^{\circ} \mathrm{C}$ ) to counteract charring of the herbs. After $5 \mathrm{~min}$, the resulting mass was poured into the mould (Clip \& Close 2.0 ice cube box, EMSA GmbH, Emsdetten, Germany) to form cubes of $10 \pm 0.1 \mathrm{~g}$. To avoid any changes of the bouillon cube, on later handling, samples for firmness measurements were prepared directly produced by pouring the hot solution into preheated (90 or $50{ }^{\circ} \mathrm{C}$ ) Petri dishes. After cooling to $20{ }^{\circ} \mathrm{C}$ all samples were stored airtight at $20 \pm 1.5^{\circ} \mathrm{C}$ for up to 6 months.

\section{Microscopy}

Light microscope images of the particles were gathered by a Zeiss Axio Scope A1 microscope equipped with an AxioCam ICm1 Rev.1 camera. Sugar crystals were administered in a SOoleogel $(10 \%(\mathrm{w} / \mathrm{w}))$ on glass microscope slides and micro- 
Table 1 Amount and type of fat type and gelator used in the variants of bouillon cubes (PO-Ref, Var 1 to Var 7)

\begin{tabular}{|c|c|c|c|c|c|c|c|c|}
\hline $\begin{array}{l}\text { Ingredients \% } \\
(\mathrm{w} / \mathrm{w})\end{array}$ & $\begin{array}{l}\text { Reference } \\
\text { (PO-Ref) }\end{array}$ & $\begin{array}{l}\text { Var } 1 \\
\text { (CO-Ref) }\end{array}$ & $\begin{array}{l}\text { Var } 2 \\
\text { (SFW_05) }\end{array}$ & $\begin{array}{l}\text { Var } 3 \\
\text { (SFW_10) }\end{array}$ & $\begin{array}{l}\text { Var 4 } \\
\text { (SO_05) }\end{array}$ & $\begin{array}{l}\text { Var 5 } \\
(\text { SO_10) }\end{array}$ & $\begin{array}{l}\text { Var } 6 \\
\text { (EC_05) }\end{array}$ & $\begin{array}{l}\text { Var } 7 \\
\text { (EC_10) }\end{array}$ \\
\hline PO & 20 & - & - & - & - & - & - & - \\
\hline SFW & - & - & 1 & 2 & - & - & - & - \\
\hline SO & - & - & - & - & 1 & 2 & - & - \\
\hline EC & - & - & - & - & - & - & 1 & 2 \\
\hline
\end{tabular}

graphs were taken from $90-5{ }^{\circ} \mathrm{C}$ with a cooling rate of $5{ }^{\circ} \mathrm{C}$ $\min ^{-1}$ using a Linkam-Stage (PE60, Labor- und AnalysenTechnik $\mathrm{GmbH}$ ). Imaging rate was $1 \mathrm{~min}^{-1}$.

\section{Colorimetry}

The colors of the bouillon cubes and the oleogels were measured using a chroma meter (CR-400 Chroma Meter; Konica Minolta, Japan) in the $L^{*} a^{*} b^{*}$ color space based on the CIE system. The $L^{*}$-value provides information about the brightness of the product. The $a^{*}$-value describes green to red tones from negative to positive values. The same systematic applies to the $b^{*}$-value which ranges from blue tones (negative) to yellow tones (positive). Calibration was performed with the associated calibration plate (No. 20633104). The color measurement of the bouillon cubes was performed in triplicate, each bouillon cube from the top and bottom side. ${ }^{30}$ Oleogels were measured from the top view in triplicate.

\section{Dispersibility}

$300 \mathrm{~g}$ distilled water were heated up to a temperature of $90{ }^{\circ} \mathrm{C}$. Once that temperature was reached a bouillon cube (10 g) was dispersed in the water. The batch was continuously stirred with an inclined blade stirrer at $500 \mathrm{rpm}$. The time until the bouillon cube was optically complete dissolved was recorded. The dispersibility of the cube was determined in triplicate in three batches each $(n=9)$.

\section{Firmness}

The firmness of the bouillon cube mass and the oleogels were measured with a zwickiLine universal testing machine (Zwick Roell Z5.0; ZwickRoell GmbH \& Co. KG; Ulm; Germany). For this purpose, a $\varnothing 2 \mathrm{~mm}$ cylinder geometry was driven into the sample until the applied force decreases by $30 \%$ starting from the maximum applied force needed to break the surface of the bouillon cube. Each composition was fabricated in triplicate. As described earlier, Petri dishes were used for firmness measurements. Previous investigations showed no influence on the measurement data with five repetitive measurements within the samples at sufficient mutual distance of $1 \mathrm{~cm}$.

\section{Sensory tests}

The bouillon cubes and their broths were tested sensorially. The cubes $(10 \mathrm{~g})$ were dissolved in $500 \mathrm{ml}$ of boiling water to produce a $2 \%(\mathrm{w} / \mathrm{w})$ broth. Before testing, the broths were allowed to cool down to $40{ }^{\circ} \mathrm{C}$. For the sensory evaluation, bouillon cubes with pure and structured CO as well as two commercial bouillon cubes: (1) Ref_1: Bio-Zentrale Naturprodukte GmbH, Wittibreut-Ulbering, Germany and (2) Ref_2: Alnatura Production and Trading GmbH, Darmstadt, Germany, were examined using triangle tests $(\mathrm{T})$ and ranking tests (R).

Table 2 shows that two triangle tests (T1 and T2) according to DIN EN ISO 4120 were carried out with a panel of 7 untrained persons each and evaluated to identify significant differences between the different broths. ${ }^{31}$ The ranking tests (R1-R5) reveals statements on consumers' acceptance. A test panel consisting of 13 persons tested oleogel-based bouillon cubes and commercially PO-based bouillon cubes (Ref_1 and Ref_2) and their broths according to the procedure above. The broths were rated from best (1) to worst (4) in the categories of: appearance and color (R1), smell (R2), taste (R3) and overall impression (R4). On top of that the bouillon cubes were evaluated in the overall impression (R5). The ranking tests were evaluated according to E DIN ISO 8587 with the Friedman test. $^{32}$

\section{Peroxide value}

For the measurement of the peroxide value (POV), the fat phase used in the formulating of the bouillon cubes was first extracted. For this, one gram of a cube was mixed in a $2: 1 \%$ $(\mathrm{v} / \mathrm{v})$ mixture of methanol and chloroform for $30 \mathrm{~min}$. The solution was filtered and concentrated in a rotary evaporator at $40{ }^{\circ} \mathrm{C}$ and $300 \mathrm{mbar}$ for $45 \mathrm{~min}$. An amount of $0.1 \pm 0.01 \mathrm{~g}$ of the extracted fat was added to $30 \mathrm{ml}$ chloroform-glacial acetic acid mixture $2: 1 \%(\mathrm{v} / \mathrm{v})$. After adding $0.5 \mathrm{ml}$ of potassium iodide solution, $30 \mathrm{ml}$ of distilled water was added to the mixture while stirring for one minute. In the next step, the measurement of the automated titrator (TitroLine 7000, SI

Table 2 Overview of the sensory tests carried out: two triangle tests of broths (T1 and T2) according to DIN EN ISO 4120 and five ranking tests of broths or bouillon cubes (R1-R5)

\begin{tabular}{lllll}
\hline $\begin{array}{l}\text { Test } \\
\text { type }\end{array}$ & type & Fat phase & Criterion & $\begin{array}{l}\text { Test } \\
\text { persons }\end{array}$ \\
\hline T1 & Broth & CO and SFW_10 & CO versus SFW & 7 \\
T2 & Broth & SO_10 and SFW_10 & SO versus SFW & 7 \\
R1 & Broth & SFW_10, Ref_1, Ref_2 & Appearance and color & 13 \\
R2 & Broth & SFW_10, Ref_1, Ref_2 & Smell & 13 \\
R3 & Broth & SFW_10, Ref_1, Ref_2 & Taste & 13 \\
R4 & Broth & SFW_10, Ref_1, Ref_2 & Overall impression & 13 \\
R5 & $\begin{array}{l}\text { Bouillon } \\
\text { cube }\end{array}$ & SFW_10, Ref_1, Ref_2 & Overall impression & 13 \\
& & &
\end{tabular}


Analytics - Xylem Analytics Germany) was used until sodium thiosulphate $(0.01 \mathrm{~mol})$ dosing resulted in equilibrium. The POV was determined in triplicate on three cubes $(n=9)$.

\section{Statistical analysis}

Statistical analysis was performed using Origin Pro 9 (OriginLab, Northhampton, MA, USA). One-way analysis of variance (ANOVA) was performed with a Tukey test using a significance level of $p \leq 0.05$. Error bars indicate standard error of mean values.

\section{Results and discussion}

\section{Appearance}

Fig. 1 shows the visual appearances of different bouillon cubes. Formulations with no gelator (upper line), PO-Ref and CO-Ref, as well as oleogel-based bouillon cube formulations (bottom lines) of SO, SFW and EC at two concentration levels, $5 \%$ and $10 \%(\mathrm{w} / \mathrm{w})$ are shown. Replacing PO with CO in the bouillon cubes resulted in prohibitive shape instabilities (Fig. 1). This illustrates clearly that $\mathrm{CO}$ alone is an inadequate lipid phase for bouillon cubes.

In contrast, all the samples studied that are based on oleogels resulted irrespective of gelator or concentration level in self-supporting, form stable bouillon cubes with similar appearance to the PO-based reference. However, significant differences between the various oleogel-formulations were detected. SO-based bouillon cubes appear darkest, have a most intense color and create the impression of a moist surface. Nevertheless, no haptic differences, such as more pronounced fatty touch, compared to the other samples was found.

SFW-based bouillon cubes look brighter than SO-based bouillon cubes and appear 'smoother' than EC-based bouillon

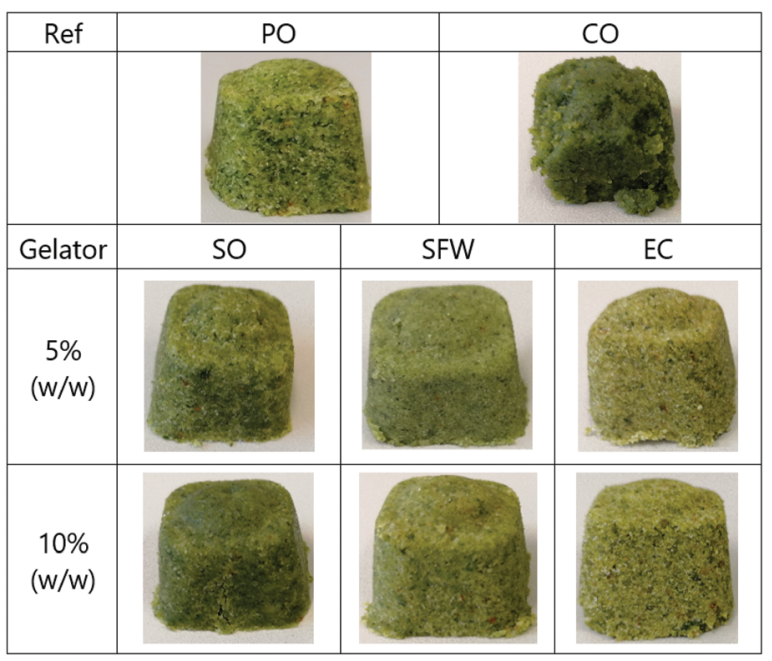

Fig. 1 Samples of the bouillon cube formulations. Upper line: PO-Ref and CO-Ref. Bottom lines: Oleogel-based bouillon cube of SO, SFW and EC on two concentration levels $5 \%$ and $10 \%(w / w)$. cubes. Nevertheless, EC-bouillon cubes have the most whitish appearance, combined with a slightly brownish tone. Additional difference to the other cubes is the optically rough appearance of the surface which seems to indicate an inhomogeneous surface structure. This effect could possibly be related to an observation made in storage time studies of the ECbased oleogels. Similarly, to the syneresis known in protein gels, the EC-based oleogels were observed to mildly contract over time. In the first place it seems fair to assume, that this phenomenon also occurs for the EC-oleogel in the context of the bouillon cube. It is however unlikely, that the complete gel phase contracts but rather that a network contraction results in oil exudation. Following this line of though one would rather expect that the oil exudated would be moving more freely and generate an oily and darker surface, contradicting the observations made in Fig. 1. Another interpretation is based on gas consumption due to oxidation. Due to the nature of the EC phase during fabrication of the bouillon cubes, the difficulty of dispersion, possibly results in more cavities. Once the gas phase is consumed due to dissolution or oxidation these voids have to be filled with oil which is sucked to the interior of the bouillon cube. Even though this observation marks an interesting starting point to look deeper into, it is considered out of scope here and will not be discussed any further. Next to this most striking observation it is noteworthy that the variation of the concentration of the gelators did practically not change the appearance of the respective bouillon cubes.

\section{Interactions gelator-particle}

In order to be able to interpret the observations made studying the different bouillon cube formulations, it is necessary to consider the interactions in the subsystems of these complex matrices. Next to the oleogel itself, the interactions between the gelators and the components of the food matrix are considered relevant.

Already previous studies have described interactions between particles and gelator. Stortz et al. demonstrated interactions between EC and different kinds of particles like sucrose and starch. The authors related those secondary effects responsible for excess hardness. ${ }^{28}$ These authors also disclosed that after fat extraction EC and sucrose are capable of forming an aerogel. The micrographs shown in Fig. 2(A-E) illustrate the interaction of gelators and sucrose particles. Micrograph F shows an EC gel with 13\% (w/w) sucrose dispersed. In this case significant changes of structure and microscopic properties were observed. For the SO structuring system, the interactions are illustrated in the series A to E. In this case the dispersion of sucrose in the gel is also exposed to higher water activity. This is known to induce the appearance of sitosterol hydrates which are in contrast to the microfibrillar structures detectable by optical microscopy. ${ }^{34}$ The images show a crystal (length $0.280 \mathrm{~cm})$ located in SO-oleogel $(10 \%$ $(\mathrm{w} / \mathrm{w}))$. The series documents temperature decreasing from $90{ }^{\circ} \mathrm{C}$ to $5^{\circ} \mathrm{C}$ with a rate of $5^{\circ} \mathrm{C} \mathrm{min}^{-1}$. From B to E the dendritic growth of sitosterol monohydrate crystals originating from 

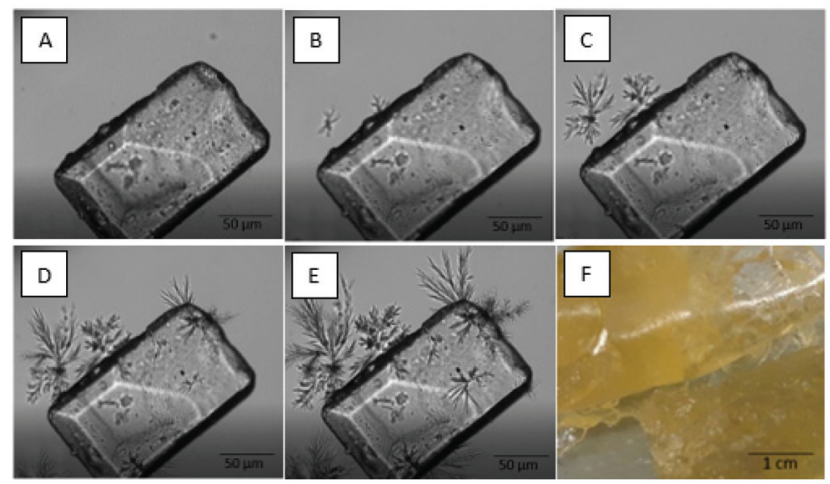

Fig. 2 (A-E) Light micrographs of a sugar crystal in a SO-oleogel melt (CO and SO $10 \%(\mathrm{w} / \mathrm{w})$ ). Temperature decreases from 90 to $50{ }^{\circ} \mathrm{C}$ with a rate of $5 \mathrm{~K} \mathrm{~min}^{-1}$. Formation of the visible oleogel-structure including the gelator-crystal-interactions. (F) Sample of $10 \%(w / w)$-EC-oleogel with $13 \%(\mathrm{w} / \mathrm{w})$ sucrose. ${ }^{33}$

the sugar crystals surface is well documented in Fig. 2. This is another clear indication that heterogeneous nucleation could play an important role in oleogel-based bouillon cubes. It has to be notes here that the superficial discussion on particle-gelatorinteractions is by no means intended to be comprehensive or properly describes the subsystems present. It is rather meant to illustrate that these interactions are not to be neglected but possibly warrant more detailed studies on the effects of surfaces, the presence of minor components and water activity in the product context. Anyhow, the observations mentioned illustrate that matrix effects on oleogel performance should not $a$ priori be neglected in specific product contexts.

\section{Colorimetry}

In support of and to quantify the impression given in Fig. 1 the $L^{*} a^{*} b$ values were determined as described above. The $L^{*} a^{*} b^{*}$-values of the oleogel-bouillon cube formulations with SFW, SO, EC (Var 2-7) and of those made with CO and PO are shown in Fig. 3A-C (left side). For reference, Fig. 3D-F (right side) illustrates the $L^{*} a^{*} b^{*}$-values of the pure fat phases used, oleogels of SFW, SO and EC at the same concentration levels. Prior to discussing individual values, the graph is briefly introduced. Absolute values and scaling can be found on the vertical axis. On the axis also, the values of the SFW wax-based samples are plotted. On the left axis values for EC-based samples are shown, on the right SO-based samples. The values of the non gelator-systems, PO and $\mathrm{CO}$ are for reasons of comparison plotted on all three dimensions. These are consequently shown as symmetric triangles. Black crosses with full black line for palm oil samples and grey crosses and lines for CO. $L^{*} a^{*} b^{*}$-values of formulations with $5 \%(\mathrm{w} / \mathrm{w})$-gelator-bouillon cubes and -oleogels are shown as black dots connected with black dotted lines. Samples with $10 \%(\mathrm{w} / \mathrm{w})$ gelator are represented as black triangles connected with black dashed lines.

Regarding Fig. 3A, brightness values ( $L^{*}$-value) of bouillon cubes, reveals that except for the cube based on the $5 \%$ SO gel the values are within a narrow range defined by the $\mathrm{CO}$ (lower bound) and the PO-based sample (upper bound). The 5\% SObased sample shows an exceptional low brightness. In general $L^{*}$-values of bouillon cubes increase with increased amount of gelator of EC and SFW $(0 \%, 5 \%$ and $10 \%(w / w))$. However, SObased bouillon cubes do not scale with concentration. For the pure fat phases investigated (Fig. 3D) the $L^{*}$-values for gels concentrate around the average value of PO and CO. The difference between the two reference systems is much bigger than found for the bouillon cubes.

It goes without saying that the mixture of herbs present in the cubes is strongly affecting the $a^{*}$ - and $b^{*}$-values (Fig. 3B and $\mathrm{C}$ ). The $a^{*}$-values (greenish) are between minus 5 and minus 15 for all bouillon cube variants (Fig. 3B). Here the CObased cubes have the highest values. PO-based cubes are least green. From the cubes based on oleogels those with EC are similar to PO, those based on SFW have values comparable to those of CO-based cubes. SO-based bouillon cubes display an intermediate green value. The $b^{*}$-values of the bouillon cubes indicate PO-based samples most yellowish. EC-based samples are close to the PO-sample. SFW-based cubes show yellowness indices in the intermediate range. Lowest values are found for CO-based cubes and those with SO oleogel. The assessment of the fat phases is obviously not influenced by the herbs and possible interaction between herbs and fat. The $a^{*}$-values are in general very low. PO and SO-based oleogels were found to be most greenish thought. Also, the $b^{*}$-values of the fat phases are relatively small and the differences between the fat phases appear to be marginal.

Comparing the results of the colorimetric measurements with impression as displayed on Fig. 1 it is striking that the variation of the values gathered is so small. Building on the hypothesis that the presence of crystals causes more reflection and hence increased whiteness can neither be found back in the visual impression (Fig. 1) nor in the experimental values (Fig. 3). Along this line of thought, one would expect a more powerful color impression of the EC- and SO-based bouillon cubes. This is so because these gelators typically form transparent gels. ${ }^{35}$

In general, the $L^{*} a^{*} b^{*}$-values of the oleogel-based formulations correspond very well to the PO-Ref. This confirms the impression from Fig. 1 that not only the appearance but especially the color of the oleogel-based products is most likely acceptable for consumers.

\section{Firmness}

To evaluate the macroscopic mechanical properties of the samples their hardness was measured as described above. Next to hardness measured also the relative hardness change (RHC) is determined. It is calculated as follows:

$$
\operatorname{RHC}(x)[\%]=\frac{F_{\max }\left(x_{\mathrm{Var}}\right)-F_{\max }\left(R e f_{\mathrm{PO}}\right)}{F_{\max }\left(R e f_{\mathrm{PO}}\right)} \times 100 \%
$$

where $F_{\max }\left(x_{\mathrm{Var}}\right)$ is the mean absolute hardness of the bouillon cube variants and $F_{\max }\left(\operatorname{Ref}_{\mathrm{PO}}\right)$ is the mean absolute hardness of the PO-Ref. With the help of the RHC values we can observe direct modifications in relation to the PO-Ref values. 


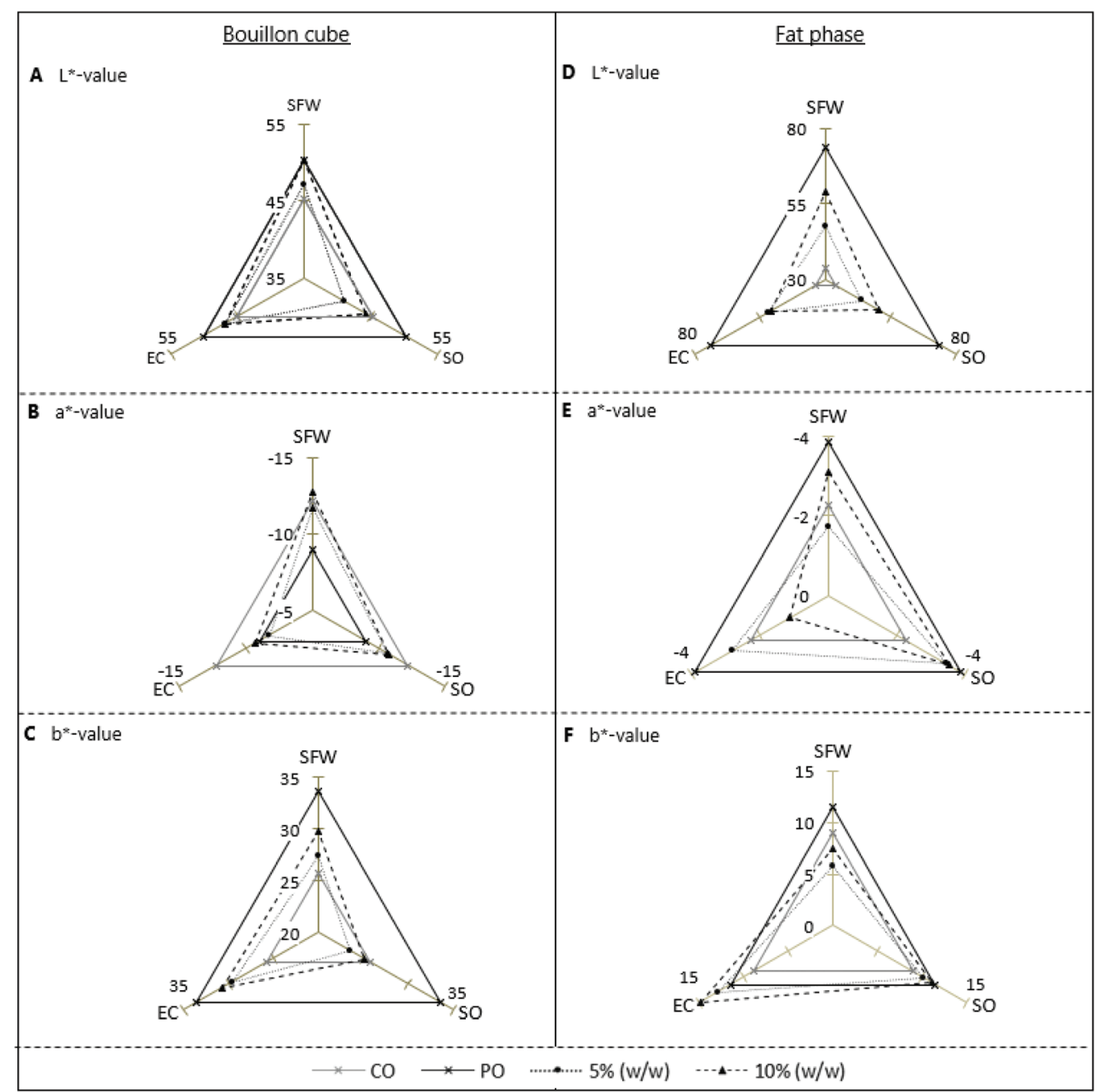

Fig. 3 (A-C) $L^{*} a^{*} b^{*}$-values of the bouillon cube formulations with CO, PO, SFW, SO and EC. (D-F) $L^{*} a^{*} b^{*}$-values of the fat phase used: pure CO and PO and oleogels of SFW, SO and EC (5\% and 10\% (w/w)). (A-F) Grey line with grey crosses: CO; black line with black crosses: PO; black dotted line with black dots: oleogel with $5 \%(\mathrm{w} / \mathrm{w})$ gelator; black dashed line with black triangles: oleogel with $10 \%(\mathrm{w} / \mathrm{w})$.

Fig. 4 shows the RHC of bouillon cubes and absolute firmness $\left(F_{\max }\right)$ values of the respective oleogels. Data originating from oleogels are illustrated in grey pillars (right-y-axis), RHCvalues of bouillon cubes in black pillars (left-y-axis). With the possibility of relating properties of the bouillon cube variants to properties of the oleogels. The gelators are plotted from left to right namely SFW, SO and EC at respective concentrations.

The $F_{\max }$-values of the oleogels (grey pillars) increases significantly with increasing gelator concentration. This is most pronounced for SO. Obviously the amount of gelators available to build a three-dimensional structure is a function of both the dosage and the solubility of the gelator in the continuous phase. The relation between gelator concentration and hardness is of interest because it allows to control final product hardness deliberately by dosage. This is discussed in more detail in another contribution of ours. ${ }^{36}$ Focussing back on the properties of the bouillon cubes it is worthwhile to compare the hardness of the cubes with the PO-Ref. The difference in

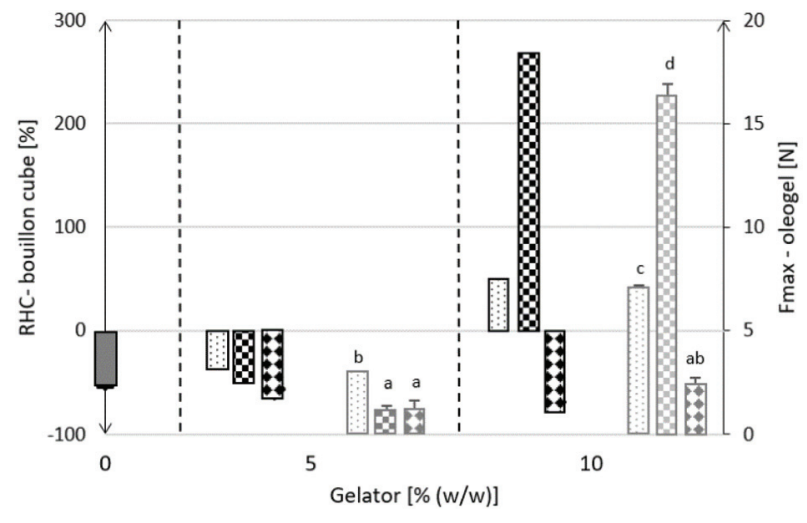

Fig. 4 RHC of bouillon cubes variants (black pillars) and $F_{\max }$ values of oleogels (grey patterned pillars) as a function of gelator concentrations, where $0 \%$ describes CO-Ref (full grey black bordered pillar). From left to right patterned pillar: SFW, SO and EC respectively. Error bars represent the standard deviation of the means while significance is indicated by the letters above the error bars (grey pillars). 
hardness from PO-Ref is expressed as RHC. These values determined are plotted as black pillars.

Substituting straight $\mathrm{CO}$ for $\mathrm{PO}$ in the bouillon cubes resulted in significant softer products (minus $47 \%$ ). For other studies, concerned with a different product microstructure, this type of substitution of the reference fat by pure $\mathrm{CO}$ was reported to lead to harder products. ${ }^{13,37,38}$ These application cannot be compared to the work here because the increased firmness is a result of better dispersed oil droplets, hence a dispersed lipid phase, in these food matrices. Unlike in these systems, the fat phase in bouillon cubes fulfills the function of integrating seasonings, more generally particles. Although, the bouillon cubes are composed of crystalline materials, the fat phase plays a major role in the physical properties of the product. Both datasets shown in Fig. 4 show at least qualitatively a surprising consistency. At $5 \%(\mathrm{w} / \mathrm{w})$ gelator dosage all cubes are softer than the reference. However, the softer gels result also in bouillon cubes with larger deviations from the reference. This trend is also found back when comparing gels based on $10 \%$ gelator dosage and the respective bouillon cubes. Harder gels result in harder bouillon cubes.

The use of $5 \%(\mathrm{w} / \mathrm{w})$ gelator resulted in softer products compared to PO-Ref, approximately minus $50 \%$. Even the hardest oleogel at $5 \%$ dosage, in this case SFW, showed only insignificant differences in firmness to the CO-Ref. At these levels of gel strength, it thus appears that the hardness of the bouillon cube is predominantly determined by the dispersed solid components. In the consideration of the cube as composite material it is important to take the density of the different components into account. This renders the volume fraction of the fat phase in excess of $30 \%$. The situation changes when harder gels, with $10 \%$ of gelator dosage in the fat phase, are used in bouillon cubes. Surprisingly, the application of a $10 \%$ EC-based oleogel results in bouillon cubes which are even softer than those based on the softer 5\% EC-based oleogel. This counterintuitive observation is possibly a result of the above-mentioned contraction of the EC-based oleogels. At the $5 \%$ EC dosage the EC containing lipid phase is possibly still rather a viscous liquid than a gel. In contrast the $10 \%$ dosage is certainly resulting in a gel. ${ }^{36}$ Consequently, could the contraction of the oleogel at $10 \%$ dosage result in a detachment of gel and dry matter due to lack of mobility of the dry matter. Even though this interpretation is rather speculative, it warrants further investigations. This observation is possibly a first indication of a feature of EC-oleogel applications that should not be neglected. For the two other gelators, SFW and SO, harder gels result in bouillon cube with significantly increased hardness. In relation to the PO-Ref, the hardness increase found is $40 \%$ for $10 \%(\mathrm{w} / \mathrm{w})$ SFW-based and $260 \%$ for $10 \%$ $(w / w)$ SO-based. The performance of the EC-based oleogels in bouillon cubes, minus $70 \%$ in hardness compared to the PO-Ref, is unexpected. This is so because known from previous work and described in the section gelator particle interaction, strong EC interactions with particles exist.

As pointed out above it is necessary to consider the solubility of the gelators in oil. Depending on the method to assess the critical composition (CGC) to form a gel the values reported are not consistent. That is due to factors such as storage temperature and time, process to initiate gelation and definition of the gel state that influence the values reported. In a recent publication the CGC was determined by extrapolation gel hardness-concentration curves to zero hardness values. ${ }^{36}$ This resulted in values of $3.8 \%, 4.6 \%$ and $9.7 \%(w / w)$ for SFW, SO and EC, respectively. In these experiments the oil used was also $\mathrm{CO}$ and the storage temperature was $22{ }^{\circ} \mathrm{C}$. Taking these CGC values into account it is not surprising that the hardness values reported for the gels in Fig. 4 are relatively small. This indicates that the bulk gel contribution is limited. Hence a hardness contribution from gelator-particle interactions seems very likely (Fig. 2). The particles of the mixture of solids, mainly composed of sodium chloride crystals with 660 microns as characteristic size, possibly agglomerate to a space filling network, with potentially solid lipid bridges contributing to the structure's hardness. It is well known, that the macroscopic properties of triglyceride based hardstock fats are highly dependent on the microstructure, where a high number of junction zones result generally in harder gels and stepwise crystallization results in excess hardness due to primary bonds. ${ }^{39}$ This structural hypothesis might appear meaningful for SFW as gelator. However, for SO and EC the nature of the three-dimensional network formed by these gelators, either a polymeric or microfibrillar network, make this interpretation less likely. These considerations indicate that elucidating the mechanism that links the gel properties to the hardness of the bouillon cubes is beyond the scope of this contribution. The hardness data on gels and bouillon cubes show nonetheless that with the gels studied gelator dosages can be found that allow to match the structural properties of the reference product.

\section{Dispersibility}

The conversion of the bouillon cube into a broth is an important quality parameter relevant for the customer's acceptance. To evaluate this the dispersibility rate in $90{ }^{\circ} \mathrm{C}$ hot water is determined for the different cubes. As shown in Fig. 1, the fat phase of the bouillon cubes is responsible for the macroscopic appearance of the products and is consequently also believed to have an influence on the dispersibility of the integrated particles. In accordance with the results obtained by Herreid et al., the dispersibility values determined in the laboratory for PO-Ref are in the range of 0.25 to $0.5 \mathrm{~g} \mathrm{~s}^{-1}$. ${ }^{40}$ The use of $\mathrm{CO}$ is in the upper range of the dispersibility rate of this range $(0.43$ $\left.\pm 0.08 \mathrm{~g} \mathrm{~s}^{-1}\right)$. The use of SO or SFW at $5 \%(\mathrm{w} / \mathrm{w})$ lead to a reduced dissolution speed. The dispersibility rate is for both type of cubes half that of the CO-Ref, $0.22 \pm 0.04 \mathrm{~g} \mathrm{~s}^{-1}$ and 0.24 $\pm 0.03 \mathrm{~g} \mathrm{~s}^{-1}$, respectively. With increasing gelator concentration to $10 \%(\mathrm{w} / \mathrm{w})$ in the fat phase of the bouillon cubes, the dispersibility rate further decreases, $0.19 \pm 0.03 \mathrm{~g} \mathrm{~s}^{-1} ; 0.19 \pm 0.03 \mathrm{~g}$ $\mathrm{s}^{-1}$, respectively. Compared to the $5 \%(\mathrm{w} / \mathrm{w})$ gelator application the decrease of only $10 \%(\mathrm{w} / \mathrm{w})$ is surprisingly low taken the changes in cube hardness into account. In all of these bouillon cube variants there is no significant decrease in dispersibility 
rate compared to the lower limit of the reference product with palm fat so that this attribute is most likely not disturbing consumer acceptance. In contrast to the results for SFW- or SObased cubes, the EC-based bouillon cubes $(5 \%$ and $10 \%(\mathrm{w} / \mathrm{w}))$ revealed strong retarding effects of the gelators on the dispersibility. Even after a duration of more than 10 minutes complete cube disintegration was not achieved. It remains difficult to unravel this observation here. However, taking that the degree of ethylation of the cellulose into account the interaction of water with EC is not surprising. After all, EC is known to stabilize w/o emulsions. ${ }^{41}$ Taking this into account turbidity due to emulsification or interaction with other solutes are actually to be expected. Consequently, this renders the application of EC for this kind of application not suitable.

\section{Sensory}

Table 3 lists the results of the evaluations of the different broths according to DIN EN ISO 412 for the triangle test and according to E DIN ISO 8587 for the ranking test. For the obvious reasons, see previous section, no broth based on ECgels was considered for this assessment. In order to detect a significant difference in the triangle tests (T1 and T2) with a certainty of $95 \%$, in the setup executed at least five test persons have to correctly identify the different samples. Table 3 shows that the number of discriminating answers is 4 out of 7 . Therefore, no significant differences were perceived between the samples tested. The sensory tests thus revealed that the use of gelled fat phases in bouillon cubes does not lead to significant organoleptic differences in the resulting broths.

Since the different prototypes (CO-, SO- and SFW-based) were indistinguishable, only SFW-bouillon cubes were taken forward as oleogel-bouillon cube in a ranking test. The $F_{\text {crit }}$ value for a probability of error of $\alpha=0.05$ of a panel of 13 is 6 (Table 3). For the ranking tests between the conventionally available (Ref_1 and Ref_2) and the oleogel-based one (SFW $10 \%(\mathrm{w} / \mathrm{w}))$ and their broths only one significant preference was found. The preference found relates to the color of the bouillon cubes. This differences in color measurements was already detected in the $L^{*} a^{*} b^{*}$-values. In the sensory test the oleogel-bouillon cubes performed with respect to color significantly better than the conventional ones. Overall, the sensory tests reveal that the bouillon cubes based on oleogels and the

Table 3 Evaluation of the results from the sensory tests (sample code see Table 2), the calculated F(E DIN ISO 8587) or D-value (DIN EN ISO $4120)$ and the respective information are given whether these calculated values are significant (YES) or not (NO)

\begin{tabular}{lllll}
\hline Code & $F$-value & $D$-value & Critical value $_{\alpha=0,05}$ & Significant difference \\
\hline T1 & - & 4 & 5 & NO \\
T2 & - & 4 & 5 & NO \\
R1 & 9.5 & - & 6 & YES \\
R2 & 4.167 & - & 6 & NO \\
R3 & 0.875 & - & 6 & NO \\
R4 & 5.542 & - & 6 & NO \\
R5 & 1.654 & - & 6 & NO
\end{tabular}

respective broths show no negative deviation from the reference.

\section{Storage stability}

In particular bouillon cubes have a long shelf life which is sometimes particularly hostile to the product due to lack of control. To reflect this, the storage stability, in detail color, dispersibility and firmness, characteristics of the bouillon cube variants were evaluated after three months storage $\left(t_{1}\right) .42$ Commercially available bouillon cubes have even longer shelf life. To cater for this the bouillon cube variants with $10 \%$ (w/w)-oleogel and CO-Ref the CO variant were additionally evaluated after six months storage $\left(t_{2}\right)$. For all storage tests the temperature was $20 \pm 1.5^{\circ} \mathrm{C}$.

Color and dispersibility of aged bouillon cubes. Potential changes of fat phases are considered critical during storage. Phenomena to be considered are oil migration, post hardening and recrystallization next to oxidation process relating to the fatty acid composition. ${ }^{42}$ Table 4 displays the development of color values and dispersibility for the different cubes studied over the shelf life. The data clearly show that for these properties no significant changes could be detected during 6 months at $20^{\circ} \mathrm{C}$. For reference the approximate values of the commercial reference product, with an undefined shelf life, are $L^{*}$-value of $53, a^{*}$-value of $-4.5, b^{*}$-value of 36 and dispersibilities between 0.28 and 0.48 .

Firmness of aged bouillon cubes. The development of the macroscopic hardness during shelf life is expressed as relative hardness change over time (RHCoT). The RHCoT helps to illustrate the changes within a variant over the respective storage period. The RHCoT is calculated as follows:

$$
\operatorname{RHCoT}[\%]=\frac{F_{\max }\left(x_{\operatorname{Var} t}\right)-F_{\max }\left(x_{\operatorname{Var} t_{0}}\right)}{F_{\max }\left(x_{\operatorname{Var} t_{0}}\right)} \times 100 \%
$$

where $F_{\max }\left(x_{\operatorname{Var} t}\right)$ is the mean maximal hardness of the bouillon cube variants at the corresponding storage time $t$ and $F_{\max }\left(x_{\operatorname{Var} t_{0}}\right)$ is the mean maximal hardness of the fresh bouillon cube variant $\left(t_{0}\right)$. Fig. 5 shows the RHCoT of the bouillon cube formulations with SFW, SO, EC and $\mathrm{CO}$ at both gelator concentrations after three months storage. Additionally, the CO-Ref and 10\% (w/w) oleogel-based bouillon cubes variants after six months storage are shown as well.

The Fig. 5 clearly illustrates that significant structural changes were observed. The CO-Ref showed the most dramatic hardness increase. Over a period of 6 months the data suggest a linear increase to a more than 4-fold increase. The question if this observation is due to rearrangement of the dispersed solids or due to minor components resulting from oil deterioration cannot be answered here. The interpretation is also hampered by the large standard deviations found indicating rather inhomogeneous structures $\left(18.1 \pm 8.6 \mathrm{~N}\left(t_{1}\right) ; 33.7 \pm 9.5\right.$ $\mathrm{N}\left(t_{2}\right)$ ). This inhomogeneity is also reflected in the inconsistent data found for dispersibility for this sample $\left(0.43\left(t_{0}\right), 0.12\left(t_{1}\right)\right.$, $\left.0.23\left(t_{2}\right)\right)$. In the interpretation of the results with respect to structural changes the sample with a $5 \%$ gelator dosage have to been treated with caution. This is so, because the sample at 


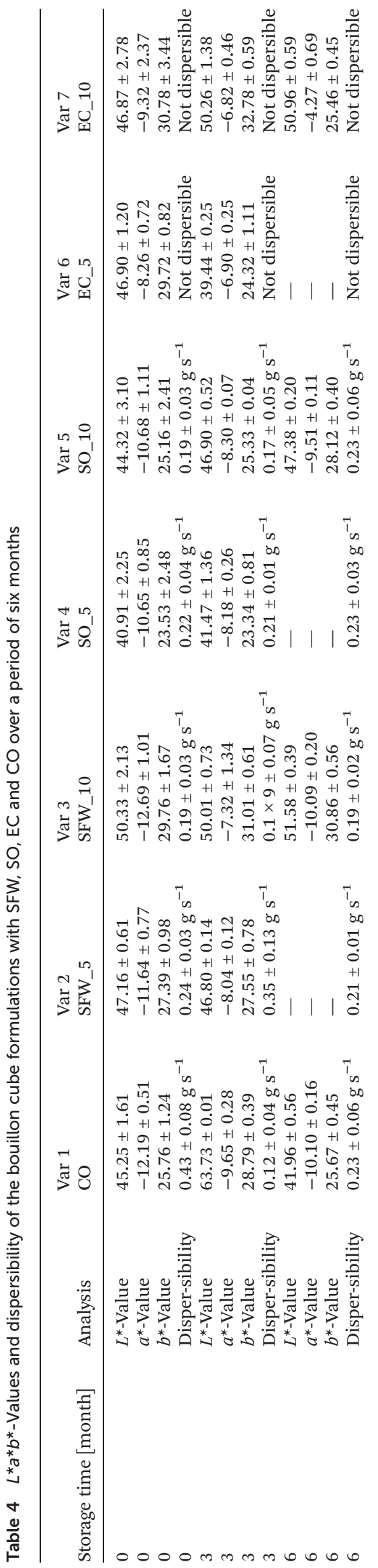

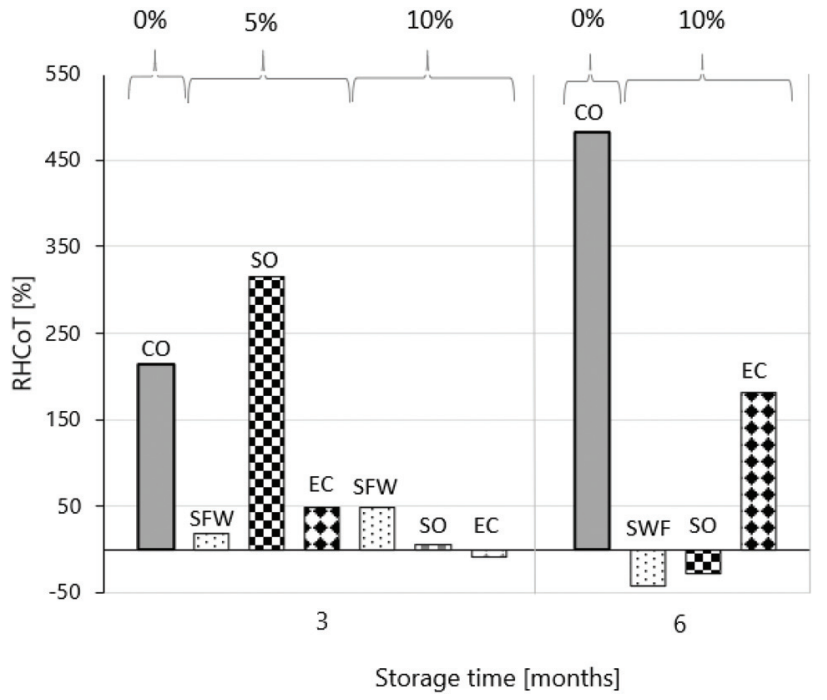

Fig. 5 RHCOT of the bouillon cube formulations with $\mathrm{CO}$ and oleogels with $5 \%$ or $10 \%(\mathrm{w} / \mathrm{w})$ of SFW, SO, EC after three months. After six months for the CO-Ref and $10 \%$ (w/w) oleogel-based bouillon cubes.

the reference point were extremely soft compromising the data on relative increase. For the bouillon cube based on EC the data suggest that the process of the hardness increase happened analogous to that in the CO sample. This is based on the fact that after 3 months the $5 \%(\mathrm{w} / \mathrm{w})$ EC sample stiffened up while the $10 \%(\mathrm{w} / \mathrm{w})$ remained practically unchanged. In this context the CGC of more than $9 \%$ should be taken into consideration. After 6 months the bouillon cube based on $10 \%(w / w)$ EC reaches an increased hardness value that falls into the range of the commercial reference product. The data on the SO-based variant deliver a more systematic picture. The sample with $5 \%$ $(\mathrm{w} / \mathrm{w})$ dosage stiffened up during the 3 months storage period. This is in line with expectation because the network formation in systems with gelator concentrations not significantly higher than the solubility is known to be slow. The $10 \%(\mathrm{w} / \mathrm{w})$ SObased samples in contrast did practically not change in the first 3 months. After 6 month these rather hard samples revealed a significant drop in hardness (minus 30\%). This can possibly be related to the well-known phenomena of conversion of the nanosized tubuli to sitosterol hydrates if the water activity is not strictly controlled. ${ }^{43}$

Lastly, the bouillon cube based on SFW-based oleogels showed the least dramatic changes. SFW has the lowest CGC so that partial crystallization of the wax can be assumed for all samples at all times. After 3 months both samples (5 and 10\% dosage) stiffened up moderately. In the following 3 months the $10 \%(\mathrm{w} / \mathrm{w})$ SFW-based cube lost about $50 \%$ of this intermediate hardness again. This loss of hardness can possibly be related to phenomena such as Ostwald ripening. In summary the hardness changes that SFW-based bouillon cubes undergo during storage appear moderate and absolute hardness values remain close to those of the commercial reference products.

Oxidation stability of aged bouillon cubes. The application oleogels in substitution scenarios in foods is often motivated 
by the elimination of partially hydrogenated fats and the simultaneous increase of unsaturated fatty acids at the expenses of saturated ones. This nutritional improvement of the fatty acid profile increases inherently the risk of oxidation and other chemical degradation of the fatty acids. Basically, vegetable oils high in unsaturated fatty acids have lower oxidative stability than fats high in saturated fatty acids. Elevated temperatures, the presence of pro-oxidative components and long storage periods increase the risk that degradation reactions progress so far that perceived product quality is negatively affected. The products evolving from oil degradation can influence perceived quality, reduce the levels of ingredients with health benefits or even lead to undesired by-products. ${ }^{44}$ Numerous studies report that oleogels are less prone to oxidation than the unstructured constituting oils. It remains however undisclosed if this is supposed to be based on any anti-oxidative action of the gelators or a possible adsorption of polar component to the dispersed phase. ${ }^{45,46}$ Some studies with extremely sensitive oils (fish origin) reported prooxidant activity of gelators though. ${ }^{47-49}$ This discussion shall not be subject of the study discussed here because the focus is the assessment of the feasibility of the oleogel application in bouillon cubes. A closer look at the ingredient list of bouillon cubes clearly reveals that this product context is hostile for oils prone to oxidation. To evaluate the extent oxidation takes place in the cubes produced here peroxide values (POV) were monitored during storage at $20 \pm 1.5{ }^{\circ} \mathrm{C}$ for up to 6 months. Samples based on EC were discarded from this assessment. This is due to the insufficient dispersibility. On one hand these gels are anyhow not fit for cube application but more so these effects were considered to corrupt the POV analytical method. To illustrate the impact of gelling, vitamin $\mathrm{E}$ addition and storage time the relative changes of the peroxide values of the investigated bouillon cube variants in relation to CO-Ref at begin of the storage period are considered. The relative peroxide change over time (RPOCoT) is introduced and calculated as follows.

$$
\operatorname{RPOCoT}[\%]=\frac{\operatorname{POV}\left(x_{\mathrm{Var} t}\right)-\operatorname{POV}\left(\mathrm{CO}_{t_{0}}\right)}{\operatorname{POV}\left(\mathrm{CO}_{t_{0}}\right)} \times 100 \%
$$

Here, $\operatorname{POV}\left(x_{\operatorname{Var} t}\right)$ is the mean POV of the bouillon cube variants at the corresponding storage time $t$ and $\operatorname{POV}\left(\mathrm{CO}_{t_{0}}\right)$ is the mean POV of the CO-bouillon-cube-variant at $t_{0}$. With the help of the RPOCoT values, direct effects depending on the CO-Ref at $t_{0}$ get visible. Fig. 6 shows the RPOCoT of the bouillon cube formulations with PO, CO, SFW and SO $(10 \%(\mathrm{w} / \mathrm{w}))$ and the formulations with added vitamin $\mathrm{E}(\mathrm{CO}+\mathrm{E}, \mathrm{SFW}+\mathrm{E}$ and $\mathrm{SO}+$ E) as a function of storage time (0, 3 and 6 months).

It is worth noting that the initial POV of the CO-Ref bouillon cubes is already above the standard $\left(<10 \mathrm{mmol} \mathrm{kg} \mathrm{g}^{-1}\right)$, which for an autocatalytic reaction most likely magnified differences. This is most likely due to the storage conditions of the $\mathrm{CO}$ which are inferior to those in industrial supply chains. As shown in Fig. 6, the POV of CO-Ref rises dramatically in the first three months of storage. Besides, it should be mentioned that the standard deviations of the means of the absolute POV

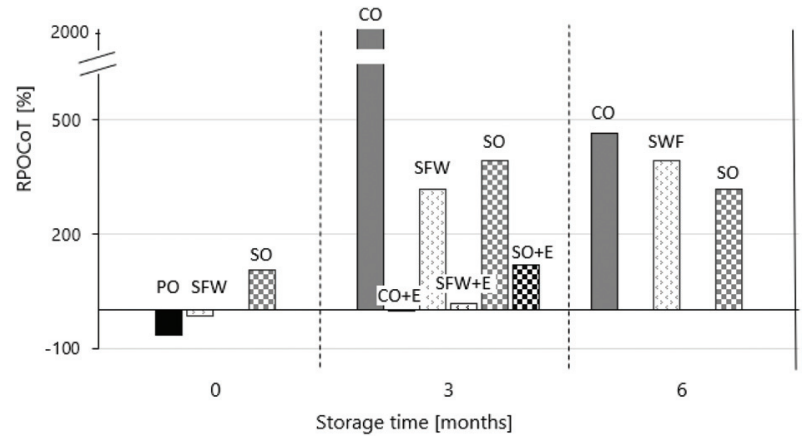

Fig. 6 RPOCoT of the bouillon cube formulations with PO, CO, 10\% $(\mathrm{w} / \mathrm{w})$ of SFW and SO over a period of six months and $10 \%(\mathrm{w} / \mathrm{w})$ of SFW, $\mathrm{SO}$ and pure $\mathrm{CO}$ plus vitamin $\mathrm{E}(\mathrm{SFW}+\mathrm{E}$; $\mathrm{SO}+\mathrm{E} ; \mathrm{CO}+\mathrm{E})$ over a period of three months.

over time are for the oleogel-based bouillon cubes and for the PO-bouillon cube (ref) small and for the CO-based bouillon cubes large. After six months of storage, the POV is significantly lower again. This is not surprising because POV characterizes and intermediate marker of deterioration.

The values gathered for fresh bouillon cubes vary significantly between the different lipid phases. The PO-Ref unsurprisingly has lowest POV levels. The SFW-based bouillon cube shows after preparation an oil quality similar to CO-Ref. The data obtained for the SO sample at $t_{0}$ are surprising and possibly caused by the preparation procedure. The elevated temperatures during the production of gels and products can influence initial oxidation states. Park et al. obtained comparable results for their model product, the cream-cheese, but attributed this to the aqueous cream-cheese network and not the oleogel network. ${ }^{7}$

After 3 months of storage the POV of the fat phase made with either SFW or SO gels are dramatically increased. Despite a three- to four-fold increase compared to the fresh CO-based cube these values were at least 5 time lower than for the stored CO-Ref. Similarly, to the observation made for the CO-Ref the POV does not increase as much during storage from 3 months to 6 months. Again, processes beyond the primary oxidation (POV) seemed to play a more dominant role in oil deterioration during this period. The observation by Lim et al. 2017 that oleogels with a harder texture have lower POV cannot be confirmed in this study and is likely limited to comparison of gels based on the same gelator. ${ }^{50}$ Hwang et al. stated that oleogels oxidise more slowly than CO due to the immobilisation of oil in the gel structure. ${ }^{47}$ Our data confirm the general observation made. But we want, as pointed out above, mention that adsorption of polar component to the structuring matrix is an alternative explanation. Along this line researches claim that oleogel applications have satisfactory oxidative stability without the addition antioxidants (AOX). ${ }^{49,51,52}$

To avoid a meaningless discussion on the level of POV that represents acceptable states of oxidation the effect of AOX addition was evaluated. For this purpose, vitamin E $(0.01 \%$ $(\mathrm{w} / \mathrm{w}))$ was added to the $10 \%$-oleogels with either SO (SO $+\mathrm{E})$ 
or $\mathrm{SFW}(\mathrm{SFW}+\mathrm{E})$ and to $\mathrm{CO}(\mathrm{CO}+\mathrm{E})$ prior to production of the bouillon cube. Vitamin $\mathrm{E}$ is known to be a potent lipid-soluble antioxidant. ${ }^{53,54}$ Fig. 6 illustrates the potency of vitamin E since for bouillon cube variants with $\mathrm{CO}+\mathrm{E}, \mathrm{SFW}+\mathrm{E}$ and $\mathrm{SO}+\mathrm{E}$ a dramatically reduced POV is measured after 3 months. For the SFW + E-based bouillon cube the value after 3 months is only mildly increased compared to the SWF-based at $t_{0}$. This is also found for the $\mathrm{CO}+\mathrm{E}$-Ref which does not change POV during the 3 months storage. Combining these data with results from the sensory assessment it is fair to conclude that at least with the application vitamin $\mathrm{E}$ no critical deterioration of the lipid phase should be expected in bouillon cubes.

\section{Conclusions}

The study presented here documents the assessment of the potential for the substitution of oleogels for highly saturated fat phases in bouillon cubes. Both technical and sensory characteristics for different product formulations were determined. For reference purposes commercial products, their palm oil-based analogues and a bouillon cube with straight canola oil were investigated next to the oleogel-based cubes. In general, all oleogel-based prototypes performed surprisingly well. The application of the ethylcellulose gel is discouraged because these bouillon cubes did not disintegrate properly in hot water. The $\beta$-Sitosterol/ $\gamma$-Oryzanol structuring system performed less good than the sunflower wax which was superior in hardness control over a 6 months shelf life. The bouillon cube utilizing straight canola oil, which was the base for all gels, were not acceptable because they cannot maintain a stable cube shape and their oxidative stability. The later could anyhow be overcome like in the other prototypes by minute addition of vitamin E. The comprehensive data gathered clearly document the feasibility to substitute oleogels for palm oil-based high SaFA fat phases in bouillon cubes. From the oleogel systems studied sunflower wax emerged as the most promising gelator.

\section{Author contributions}

VC conceived and designed the experiments and the concept and wrote the paper. ST collected the data. EF supervised the research. All authors read and approved the final manuscript.

\section{Conflicts of interest}

There are no conflicts of interest to declare.

\section{Acknowledgements}

This research was funded by the German Federal Ministry of Education and Research for the Competence Cluster NutriAct Nutrition Research Berlin-Potsdam, Germany (FKZ: 01EA1806C).

\section{References}

1 W. Hamm, R. J. Hamilton and G. Calliauw, Edible oil processing, John Wiley \& Sons, Ltd, Hoboken, NJ, USA, 2013.

2 N. M. de Roos, M. L. Bots and M. B. Katan, Replacement of Dietary Saturated Fatty Acids by Trans Fatty Acids Lowers Serum HDL Cholesterol and Impairs Endothelial Function in Healthy Men and Women, Arterioscler. Thromb. Vasc. Biol., 2001, 21, 1233-1237.

3 R. Mensink, P. Zock, A. Kester and M. B. Katan, Effects of dietary fatty acids and carbohydrates on the ratio of serum total to HDL cholesterol and on serum lipids and apolipoproteins: a meta-analysis of 60 controlled trials, Am. J. Clin. Nutr., 2003, 77, 1146-1155.

4 World Health Organization, Guidelines: Saturated Fatty Acid and Trans-Fatty Acid Intake for Adults and Children (draft issued for public consultation in May 2018), 2018.

5 A. Wendt, K. Abraham, C. Wernecke, J. Pfeiffer and E. Flöter, Application of $\beta$-Sitosterol $+\gamma$-Oryzanol-Structured Organogel as Migration Barrier in Filled Chocolate Products, J. Am. Oil Chem. Soc., 2017, 94, 1131-1140.

6 H. L. Bemer, M. Limbaugh, E. D. Cramer, W. J. Harper and F. Maleky, Vegetable organogels incorporation in cream cheese products, Food Res. Int., 2016, 85, 67-75.

7 C. Park, H. L. Bemer and F. Maleky, Oxidative Stability of Rice Bran Wax Oleogels and an Oleogel Cream Cheese Product, J. Am. Oil Chem. Soc., 2018, 95, 1267-1275.

8 D. C. Zulim Botega, A. G. Marangoni, A. K. Smith and H. D. Goff, The Potential Application of Rice Bran Wax Oleogel to Replace Solid Fat and Enhance Unsaturated Fat Content in Ice Cream, J. Food Sci., 2013, 78, 1334-1339.

9 H.-S. Hwang, M. Singh, J. K. Winkler-Moser, E. L. Bakota and S. X. Liu, Preparation of margarines from organogels of sunflower wax and vegetable oils, J. Food Sci., 2014, 79, C1926-C1932.

10 T. A. Stortz and A. G. Marangoni, Ethylcellulose solvent substitution method of preparing heat resistant chocolate, Food Res. Int., 2013, 51, 797-803.

11 S. Barbut, J. Wood and A. Marangoni, Potential use of organogels to replace animal fat in comminuted meat products, Meat Sci., 2016, 122, 155-162.

12 J. Gómez-Estaca, A. M. Herrero, B. Herranz, M. D. Álvarez, F. Jiménez-Colmenero and S. Cofrades, Characterization of ethyl cellulose and beeswax oleogels and their suitability as fat replacers in healthier lipid pâtés development, Food Hydrocolloids, 2019, 87, 960-969.

13 A. K. Zetzl, A. G. Marangoni and S. Barbut, Mechanical properties of ethylcellulose oleogels and their potential for saturated fat reduction in frankfurters, Food Funct., 2012, 3, 327-337.

14 F. Caponio, T. Gomes and M. T. Bilancia, Bouillon cubes: assessment of the state of degradation of the lipid fraction, J. Sci. Food Agric., 2003, 83, 1331-1336.

15 E. O. Akpanyung, Proximate and mineral element composition of bouillon cubes produced in Nigeria, Pak. J. Nutr., 2005, 4, 327-329. 
16 Z. Chen and W. Oldewage-Theron, Household consumption of stock cubes and stock powder in the Vaal Triangle of SA, Nutr. Food Sci., 2004, 34, 174-178.

17 J. R. Ham, Cooking to be Modern but Eating to be Healthy: The Role of Dawa-Dawa in Contemporary Ghanaian Foodways, Food Cult. Soc., 2017, 20, 237-256.

18 D. Moretti, R. F. Hurrell and C. I. Cercamondi, Food Fortification in a Globalized World, Bouillon Cubes, 2018.

19 F. Rohner, M. Leyvraz, A. G. Konan, L. J. C. E. Esso, J. P. Wirth, A. Norte, A. F. Adiko, B. Bonfoh and G. J. Aaron, The Potential of Food Fortification to Add Micronutrients in Young Children and Women of Reproductive Age Findings from a Cross-Sectional Survey in Abidjan, Côte d'Ivoire, PLoS One, 2016, 11, 1-13.

20 F. Staubli Asobayire, Development of a food fortification strategy to compat iron deficiency in the Ivory Coast, 2000.

21 S. Gupta and P. Bongers, Bouillon cube process design by applying product driven process synthesis, Chem. Eng. Process., 2011, 50, 9-15.

22 F. Caponio, T. Gomes and D. Delcuratolo, Qualitative and quantitative characterisation of the lipid fraction of bouillon cubes, Eur. Food Res. Technol., 2002, 215, 200203.

23 L. Karsulinova, B. Folprechtova, M. Dolezal, J. Dostalova and J. Velisek, Analysis of the Lipid Fractions of Coffee Creamers, Cream Aerosols, and Bouillon Cubes for Their Health Risk Associated Constituents, Czech J. Food Sci., 2007, 25, 257-264.

24 A. I. Blake, E. D. Co and A. G. Marangoni, Structure and Physical Properties of Plant Wax Crystal Networks and Their Relationship to Oil Binding Capacity, J. Am. Oil Chem. Soc., 2014, 91, 885-903.

25 T. Wettlaufer, B. Hetzer and E. Flöter, Characterization of Oleogels based on waxes and their hydrolyzates, Eur. J. Lipid Sci. Technol., 2021, 2000345.

26 A. Bot and W. G. M. Agterof, Structuring of Edible Oils by Mixtures of $\gamma$-Oryzanol with $\beta$-Sitosterol or Related Phytosterols, JAOCS, 2006, 2006, 513-521.

27 A. Bot and E. Flöter, in Edible Oleogels, Elsevier, 2018, pp. 31-63.

28 T. A. Stortz, D. C. de Moura, T. Laredo and A. G. Marangoni, Molecular interactions of ethylcellulose with sucrose particles, RSC Adv., 2014, 4, 55048-55061.

29 M. Scharfe and E. Flöter, Oleogelation: From Scientific Feasibility to Applicability in Food Products, Eur. J. Lipid Sci. Technol., 2020, 2000213, 1-24.

30 N. Ismail and N. S. Sahibon, Evaluation of Bouillon Cube Prepared with the Addition of Threadfin Bream (Nemipterus japonicas) Hydrolysate, Pertanika J. Trop. Agric. Sci., 2018, 41, 1315-1328.

31 Sensory analysis - Methodology - Triangle test (ISO 4120:2004); German version EN ISO 4120:2007, Oktober 2007, ICS 67.240.

32 Sensory analysis - Methodology - Ranking (ISO 8587:2006), August 2010, ICS 67.240.
33 J. Swiacka, Bachelor Thesis: Interaction of sucrose and oleogel - gel properties and organoleptics, Berlin, Germany, Technical University Berlin, 2019.

34 H. Sawalha, R. den Adel, P. Venema, A. Bot, E. Flöter and E. van der Linden, Organogel-emulsions with mixtures of $\beta$-sitosterol and $\gamma$-oryzanol: influence of water activity and type of oil phase on gelling capability, J. Agric. Food Chem., 2012, 60, 3462-3470.

35 A. B. Matheson, V. Koutsos, G. Dalkas, S. Euston and P. Clegg, Microstructure of $\beta$-Sitosterol: $\gamma$-Oryzanol Edible Organogels, Langmuir, 2017, 33, 4537-4542.

36 E. Flöter, T. Wettlaufer, V. Conty and M. Scharfe, OleogelsTheir Applicability and Methods of Characterization, Molecules, 2021, 26, 1-19.

37 E. D. Paneras and J. G. Bloukas, Vegetable Oils Replace Pork Backfat for Low-Fat Frankfurters, J. Food Sci., 1994, 59, 725-728.

38 M. K. Youssef and S. Barbut, Effects of protein level and fat/oil on emulsion stability, texture, microstructure and color of meat batters, Meat Sci., 2009, 82, 228-233.

39 K. Sato, Crystallization of Lipids, John Wiley \& Sons, Ltd, Hoboken, NJ, USA, 2018.

40 R. M. Herreid and V. E. Lippert, Method for Making Fast Dissolving Bouillon Cubes, United States Patent, 6126979, 2000.

41 E. Melzer, J. Kreuter and R. Daniels, Ethylcellulose: a new type of emulsion stabilizer, Eur. J. Pharm. Biopharm., 2003, 56, 23-27.

42 R. Raitio, V. Orlien and L. H. Skibsted, Effects of palm oil quality and packaging on the storage stability of dry vegetable bouillon paste, Food Chem., 2012, 132, 13241332.

43 A. Bot, Y. S. Veldhuizen, R. den Adel and E. C. Roijers, NonTAG structuring of edible oils and emulsions, Food Hydrocolloids, 2009, 23, 1184-1189.

44 A. Kaleem, S. Aziz, M. Iqtedar, R. Abdullah, M. Aftab, F. Rashid, F. R. Shakoori and S. Naz, Investigating Changes and Effect of Peroxide Values in Cooking Oils Subject to Light and Heat, FUUAST J. Biol., 2015, 5, 191-196.

45 M. Scharfe, J. Niksch and E. Flöter, Influence of minor oil components on sunflower, rice bran, candelilla and beeswax oleogels, Eur. J. Lipid Sci. Technol., 2021, Under review.

46 M. Scharfe, Y. Ahmane, J. Seilert, J. Keim and E. Flöter, On the Effect of Minor Oil Components on $\beta$-Sitosterol $/ \gamma$-oryzanol Oleogels, Eur. J. Lipid Sci. Technol., 2019, 121, 1-13.

47 H.-S. Hwang, M. Fhaner, J. K. Winkler-Moser and S. X. Liu, Oxidation of Fish Oil Oleogels Formed by Natural Waxes in Comparison With Bulk Oil, Eur. J. Lipid Sci. Technol., 2018, 120, 1700378.

48 B. Yi, M.-J. Kim, S. Y. Lee and J. Lee, Physicochemical properties and oxidative stability of oleogels made of carnauba wax with canola oil or beeswax with grapeseed oil, Food Sci. Biotechnol., 2017, 26, 79-87.

49 E. Yılmaz, M. Öğütcü and Y. K. Yüceer, Physical Properties, Volatiles Compositions and Sensory Descriptions of the 
Aromatized Hazelnut Oil-Wax Organogels, J. Food Sci., 2015, 80, 2035-2044.

50 J. Lim, H.-S. Hwang and S. Lee, Oil-structuring characterization of natural waxes in canola oil oleogels: rheological, thermal, and oxidative properties, Appl. Biol. Chem., 2017, 60, 17-22.

51 T. L. T. Da Silva, K. F. Chaves, G. D. Fernandes, J. B. Rodrigues, H. M. A. Bolini and D. B. Arellano, Sensory and Technological Evaluation of Margarines With Reduced Saturated Fatty Acid Contents Using Oleogel Technology, J. Am. Oil Chem. Soc., 2018, 95, 673-685.
52 B. M. Ferrer-González, I. García-Martínez and A. Totosaus, Textural properties, sensory acceptance and fatty acid profile of cooked meat batters employing pumpkin seed paste or soybean oil oleogel as fat replacers, Grasas Aceites, 2019, 70, e320.

53 Antioxidants in food, ed. J. Pokorny, Woodhead; CRC Press, Cambridge, Boca Raton, Fla., 2003.

54 D. J. Buckley, P. A. Morrissey and J. I. Gray, Influence of dietary vitamin $\mathrm{E}$ on the oxidative stability and quality of pig meat, J. Anim. Sci., 1995, 73, 31223130 . 